\title{
Preface to the special topic on tissue stem cell research
}

\author{
Jinsong $\mathrm{Li}^{1,2^{*}} \&$ Yi Arial Zeng ${ }^{1,2^{*}}$ \\ ${ }^{1}$ State Key Laboratory of Cell Biology, Shanghai Institute of Biochemistry and Cell Biology, CAS Center for Excellence in Molecular Cell \\ Science, Chinese Academy of Sciences, Shanghai 200031, China; \\ ${ }^{2}$ School of Life Science, Hangzhou Institute for Advanced Study, University of Chinese Academy of Sciences, Chinese Academy of Sciences, \\ Hangzhou 310024, China
}

Received October 23, 2021; published online November 30, 2021

Citation: Li, J., and Zeng, Y.A. (2021). Preface to the special topic on tissue stem cell research. Sci China Life Sci 64, 1995-1997. https://doi.org/10.1007/ s11427-021-2012-1

Stem cells have the ability to self-renew, divide for a long period of time, and differentiate into specialized cells with distinct functions. Scientists are hopeful that, in the future, stem cells will be used to cure a wide range of diseases and traumatic injuries with some application of cell therapy. Currently, donated organs and tissues are used to replace lost or damaged tissue in many disorders. However, we do not have enough organs. How can we resolve the cell limitation and provide a solution that could help more patients not just to select few? The great regenerative potential of stem cells has created intense research interest. Patient-originated stem cell-based cell replacement therapy has become a new pillar of human medicine (Fischbach et al., 2013). Especially, through combining with CRISPR-Cas9-mediated gene corrections or modifications, it enables personalized treatment of genetic disorders (Yan and Li, 2020) or derivation of nextgeneration of stem cells with enhanced features and functionalities for treatment of intractable diseases, such as tumors (Kimbrel and Lanza, 2020). There are two kinds of patient-originated stem cells, i.e., pluripotent stem cells (PSCs) and naturally occurring tissue stem cells (also referred to as adult stem cells). Induced PSCs (iPSCs) can be readily derived from patient's somatic cells through transient overexpression of Yamanaka factors. However, genetic instability occurs during nuclear reprogramming induced by

*Corresponding authors (Jinsong Li, email: jsli@sibcb.ac.cn; Yi Arial Zeng, email: yzeng@sibcb.ac.cn)
Yamanaka factors (Jiang et al., 2013), resulting in cells with genetic defects that may cause tumorigenesis. Meanwhile, somatic cell nuclear transfer can be also used to generate nuclear transfer embryonic stem cells (ntESCs) from patients. However, the low efficiency of ntESC generation and the ethical concerns on destroying human embryos are two bottlenecks of its application. In contrast, tissue stem cells (TSCs) that sustain multipotent or unipotent are less tumorigenicity. TSCs are rare, undifferentiated cells present in many adult tissues. The primary role of TSCs is to differentiate into functional somatic cells to maintain and repair the tissue in which they reside (Fuchs and Blau, 2020). Therefore, TSCs if can be isolated and stably maintained in vitro can be theoretically safer cell resources for therapeutic applications. However, major challenges for developing therapies based on TSCs are whether the specific tissues/ organs have TSCs and how to harvest and culture them in vitro. With the application of advanced technologies, such as single-cell RNA sequencing (scRNA-seq), next-generation lineage tracing, live tissue imaging, organoid culture, and CRISPR-Cas9-mediated genome-editing, a couple of new TSCs have been identified and expanded in vitro in recent years.

In the past two decades, with the continuous funding support from the Chinese Academy of Sciences (CAS), the National Natural Science Foundation (NSFC), and the Ministry of Science and Technology (MOST) on the fields of stem cell and development (Yao et al., 2013), Chinese sci- 
entists have played a significant role in promoting the development of the TSC field by making enormous achievements, especially in the last five years. To reflect the recent advances in TSC field in China, we present our readers the specific topic on "tissue stem cell research" with five highquality, peer-reviewed articles, including four reviews and one original research article.

Identification, validation, isolation, and expansion of TSCs are the first steps of their clinical applications. In a comprehensive review article by Ye-Guang Chen, Dong Gao, Ping Hu, Lijina Hui, Xiaoqun Wang, Yi Zeng and colleagues discussed the recent progress in TSCs from various tissues, including the brain, muscle, liver, pancreatic islet, intestine, and prostate (Fu et al., 2021). ScRNA-seq has been employed in all these tissues/organs for deciphering cellular heterogeneity and uncovering rare cell types including TSCs, such as two recent reports on the identification of pancreatic islet TSCs and prostate TSCs respectively (Guo et al., 2020; Wang et al., 2020). Further validation of their stemness in vivo was analyzed by linage tracing strategies along with the generation of various genetic mouse models. Importantly, the use of organoid culture has enabled long-term maintenance of adult TSCs from the pancreas and prostate in vitro. Meanwhile, long-term expansion of mouse muscle stem cells (MuSCs) in vitro has been successfully achieved by mimicking the endogenous microenvironment of muscle injury that inflammation signals and myofiber necroptosis releases factors to activate MuSCs for muscle regeneration (Fu et al., 2015; Zhou et al., 2020). The adult lung is one of the most highly stressed organs in our body due to its function of gas exchange between the blood and the external environment. Thus, it is critical to maintain the lung's homeostasis. The review article by Yanxiao Wang and Nan Tang provides an overview of the known TSCs/progenitor cells, their subpopulations, and the regulatory networks to maintain their stemness and differentiation potentials in different regions of the adult lung under certain physiological and pathological conditions (Wang and Tang, 2021). Although the microenvironment of TSCs is dramatically different among tissues/organs, TSCs sustain similar paradigms regarding their physiological features. The review article by Tao Cheng and colleagues describes a "SMART" model to delineate the key characteristics of TSCs, including self-renewal, multi-lineage differentiation, apoptosis, rest, and trafficking, based on the knowledge of hematopoietic stem cells (HSCs) (Yuan et al., 2021), which may be used to speculate on the physiological relevance of TSCs in other tissues/organs. HSCs with adult-reconstituting multilineage potential are believed to originate from both intraembryonic and extraembryonic regions. While the intraembryonic origin of HSCs is well supported by a large number of studies, the extraembryonic yolk sac (YS) origin of HSCs remains controversial. The research article by Yu Lan and colleagues reports that YS- originated endothelial cells sustain the HSC potential by showing short-term myeloid and lymphoid lineage repopulating capacity after transplantation into recipient mice (Wang et al., 2021). Obviously, advanced technologies such as genetic lineage tracing contribute greatly to the recent advances in TSC field. Genetic lineage tracing can accurately track cell movement, proliferation, differentiation, and cell fate switching in vivo, thus enabling deciphering the cell fate of TSCs during development, tissue/organ repair, and regeneration. The technology review article by Bin Zhou and colleagues describe the overview of the cell lineage tracing methods and summarize the next-generation lineage tracing strategy established based on dual recombinases that can be used to study TSC origin and fate in vivo with improved both the resolution and precision (Jin et al., 2021).

The scientific advances in TSC field in China greatly benefit from the rapid progress of new technologies and stably increased funding support on basic research during the past years. With these achievements, we are optimistic and confident that the successful trend of TSC research will continue for the next decades in not only basic research but also translational research, which will greatly promote therapeutic applications of TSCs. Finally, as the guest editors, we would like to greatly appreciate all the authors, reviewers, and also the editorial staff of SCLS for their contributions to make this special topic possible. We hope that this special topic will receive broad attention from the researchers and will contribute to the growth of TSC biology in years to come.

\section{References}

Fischbach, M.A., Bluestone, J.A., and Lim, W.A. (2013). Cell-based therapeutics: the next pillar of medicine. Sci Transl Med 5, 179ps177.

Fu, X., He, Q., Tao, Y., Wang, M., Wang, W., Wang, Y., Yu, Q.C., Zhang, F., Zhang, X., Chen, Y.G., et al. (2021). Recent advances in tissue stem cells. Sci China Life Sci 64, 1998-2029.

Fu, X., Xiao, J., Wei, Y., Li, S., Liu, Y., Yin, J., Sun, K., Sun, H., Wang, H., Zhang, Z., et al. (2015). Combination of inflammation-related cytokines promotes long-term muscle stem cell expansion. Cell Res 25, 655-673.

Fuchs, E., and Blau, H.M. (2020). Tissue stem cells: architects of their niches. Cell Stem Cell 27, 532-556.

Guo, W., Li, L., He, J., Liu, Z., Han, M., Li, F., Xia, X., Zhang, X., Zhu, Y., Wei, Y., et al. (2020). Single-cell transcriptomics identifies a distinct luminal progenitor cell type in distal prostate invagination tips. Nat Genet 52, 908-918.

Jiang, J., Lv, W., Ye, X., Wang, L., Zhang, M., Yang, H., Okuka, M., Zhou, C., Zhang, X., Liu, L., et al. (2013). Zscan4 promotes genomic stability during reprogramming and dramatically improves the quality of iPS cells as demonstrated by tetraploid complementation. Cell Res 23, 92106.

Jin, H., Liu, K., and Zhou, B. (2021). Dual recombinases-based genetic lineage tracing for stem cell research with enhanced precision. Sci China Life Sci 64, 2060-2072.

Kimbrel, E.A., and Lanza, R. (2020). Next-generation stem cells-ushering in a new era of cell-based therapies. Nat Rev Drug Discov 19, 463-479.

Wang, C., Gong, Y., Wei, A., Huang, T., Hou, S., Du, J., Li, Z., Wang, J., 
Liu, B., and Lan, Y. (2021). Adult-repopulating lymphoid potential of yolk sac blood vessels is not confined to arterial endothelial cells. Sci China Life Sci 64, 2073-2087.

Wang, D., Wang, J., Bai, L., Pan, H., Feng, H., Clevers, H., and Zeng, Y.A. (2020). Long-term expansion of pancreatic islet organoids from resident Procr+ progenitors. Cell 180, 1198-1211.e19.

Wang, Y., and Tang, N. (2021). The diversity of adult lung epithelial stem cells and their niche in homeostasis and regeneration. Sci China Life Sci 64, 2045-2059.

Yan, M., and Li, J. (2020). Combined application of CRISPR-Cas and stem cells for clinical and basic research. Cell Regen 9, 19.

Yao, X., Li, D., and Pei, G. (2013). In focus: molecular and cell biology research in China. Nat Rev Mol Cell Biol 14, 600-606.

Yuan, S., Sun, G., Zhang, Y., Dong, F., Cheng, H., and Cheng, T. (2021). Understanding the "SMART" features of hematopoietic stem cells and beyond. Sci China Life Sci 64, 2030-2044.

Zhou, S., Zhang, W., Cai, G., Ding, Y., Wei, C., Li, S., Yang, Y., Qin, J., Liu, D., Zhang, H., et al. (2020). Myofiber necroptosis promotes muscle stem cell proliferation via releasing Tenascin-C during regeneration. Cell Res 30, 1063-1077.

\section{Biographical Sketch}
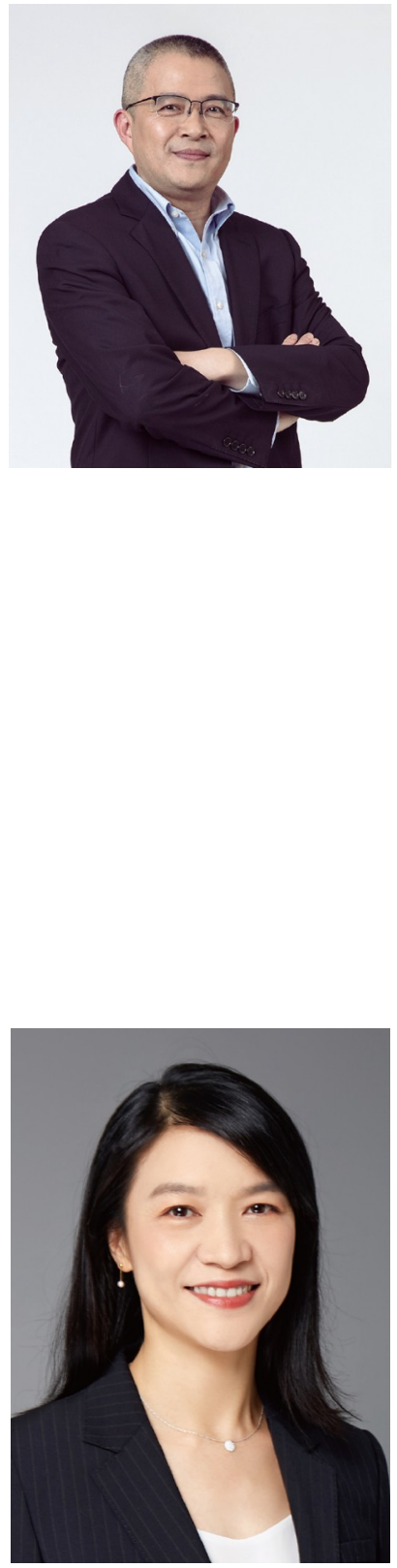

Jinsong Li is a professor and director of State Key Laboratory of Cell Biology, the Shanghai Institute of Biochemistry and Cell Biology (SIBCB), Chinese Academy of Sciences (CAS). He obtained his $\mathrm{PhD}$ from Institute of Zoology, CAS, in 2002 and followed by postdoctoral training at Rockefeller University before joining SIBCB in 2007. His research mainly focuses on stem cells and embryonic development. He has made fundamental contributions to the establishment of androgenetic haploid embryonic stem cells (also termed "sperm-like stem cells") that can be used as sperm replacement for efficient production of semi-cloned mice (so called semi-cloning (SC) technology). He has made great efforts to promote the applications of $\mathrm{SC}$ technology and shown that it can be used as a unique tool for genetic analyses in mice, including efficient generation of mouse models carrying defined point mutations related to human developmental defects; one-step generation mouse models that mimic multiple genetic defects in human diseases; and medium-scale targeted screening of critical genes or critical nucleotides of a specific gene involved in a developmental process. Most recently, he initiated and is promoting a huge project to tag every protein in mice based on "sperm-like stem cell"mediated SC technology (genome tagging project, GTP), which may enable the precise description of protein expression and localization patterns, and protein-protein, protein-DNA and protein-RNA interactions in development/aging, physiological and pathological conditions. He is a recipient of the "100 talents project" of CAS, National Science Fund for Excellent Young Scholars, A-IMBN Research Young Investigators Award, Tan Jiazhen Life Science Innovation Award, Ho Leung Ho Lee Foundation Innovation Award, CSCB Outstanding Accomplishment Award, and Guanghua Engineering Science and Technology Prize. He currently serves as the editor-in-chief for Asian J Andro, associate editor for $J M C B, J G G$, and Cell Regen, and on the editorial board of JBC, Open Biol, Biol Reprod, and Sci China Life Sci.

Yi Zeng is a Principal Investigator at the Institute of Biochemistry and Cell Biology, Chinese Academy of Sciences. She did her PhD at Simon Fraser University in Canada and postdoctoral training at Stanford University. Her research interest is to understand the regulatory mechanisms of adult stem cells in various tissues, and the interaction between stem cells and their niche. She has focused her efforts on Procr (protein $\mathrm{C}$ receptor), which has been established as a surface marker of adult stem cells in multiple tissues, including the mammary gland, the endothelium, the hematopoietic system, and the ovarian epithelium. Her recent work has identified the long-sought pancreatic islet stem cells. The goal of her research is to determine how the stem cell regulatory mechanisms have deviated in aging and diseases, to learn how to activate the stem cells in situ, as well as to provide ever-expanding stem cells in vitro for regenerative medicine. She is a recipient of the Young Investigator Award of Chinese Society for Cell Biology (2015), National Science Fund for Excellent Young Scholars (2016), Tan Jiazhen Life Science Innovation Award (2020) and Xplorer Prize (2021). She currently serves as an editor for eLife and Differentiation, and on the editorial board of Development. 